\title{
DESENVOLVIMENTO DE PÃO DE QUEIJO COM TEOR REDUZIDO DE SÓDIO
}

Telma Reginato Martins, Andréia de Menezes Olivo, Keli Maria Consoli

Universidade do Oeste Paulista - UNOESTE. Pós-graduação em Processamento de Alimentos, Presidente Prudente SP. E-mail: telma@unoeste.br

\begin{abstract}
RESUMO
É crescente a preocupação com a saúde da população da sociedade moderna, especialmente no que tange a hábitos de vida saudável e alimentação. A ingestão de sal em excesso é comprovadamente um fator de risco para doenças cardiovasculares relacionadas ao aumento da pressão arterial. O pão de queijo é um produto tradicionalmente brasileiro, obtido da mistura de polvilho com água ou leite, queijo, ovos, sal e gordura. Esta pesquisa teve como objetivo desenvolver uma formulação de pão de queijo com teor reduzido de sódio, visando a obtenção de um produto funcional e ao mesmo tempo saboroso e aceitável, mediante avaliação sensorial em escala hedônica de 9 pontos com equipe não treinada de 50 provadores. Ao avaliar os testes de aceitabilidade global aplicados no pão de queijo convencional e no produto com redução de sódio, observou-se que ambas formulações apresentaram notas médias similares (7,85 para o pão de queijo tradicional e 7,75 para o pão de queijo com teor reduzido em sódio). 0 resultado das análises estatísticas demonstra que esta diferença não foi significativa ao nível $5 \%$ de probabilidade $(p<0,05)$.
\end{abstract}

Palavras-chave: pão de queijo; cloreto de sódio; pressão arterial; alimento funcional.

\section{DEVELOPMENT OF CHEESE BREAD WITH LOW SODIUM CONTENT}

\begin{abstract}
There is growing concern about the health of the population of modern society, especially in regard to healthy lifestyle habits and diet. Salt intake in excess is a proven risk factor for cardiovascular diseases related to increased blood pressure. The cheese bread is traditionally a Brazilian product, obtained by mixing starch with water or milk, cheese, eggs, salt and fat. This research aimed to develop a formulation of cheese bread with low sodium content, in order to obtain a functional product while tasty and acceptable by sensory evaluation in hedonic 9-point scale with 50 staff not trained tasters. In assessing the overall acceptability tests applied in conventional bread and cheese product with reduced sodium, it was found that both formulations had similar average notes ( 7.85 to traditional bread and cheese to 7.75 cheesebread with low sodium content). The result of statistical analysis demonstrates that this difference was not significant at $5 \%$ probability $(p<0.05)$.
\end{abstract}

Keywords: cheese bread; sodium chloride; blood pressure; functional food. 


\section{INTRODUÇÃO}

Atualmente os consumidores têm atribuído grande importância aos aspectos que afetam diretamente a sua qualidade de vida. A dieta, apesar de não ser o único fator que afeta a saúde e o bem-estar, é dos mais importantes. Logo, é natural que na sociedade atual exista, cada vez mais, maior procura por produtos alimentares mais saudáveis. Para serem catalogados como tal, estes produtos têm que apresentar pelo menos uma das seguintes características: composição alterada e/ou condições de processamento que previnam ou limitem a presença de determinado composto potencialmente perigoso, e/ou a possibilidade de inclusão de substâncias desejáveis, quer naturais, quer por adição, com os subsequentes benefícios para a saúde (ORVALHO, 2010).

Para Sarno et al. (2009) são várias as evidências que relacionam o consumo excessivo de sal ao desenvolvimento de doenças crônicas. Estima-se que, entre 25 e 55 anos de idade, uma diminuição de apenas 1,3 g na quantidade de sódio consumida diariamente se traduziria em redução de 5 $\mathrm{mmHg}$ na pressão arterial sistólica ou de $20 \%$ na prevalência de hipertensão arterial. Além disso, haveria também substanciais reduções na mortalidade por acidentes vasculares cerebrais (14\%) e por doença coronariana (9\%), representando 150.000 vidas salvas anualmente em todo o mundo. O sódio é adicionado sob a forma de cloreto de sódio (composto por $40 \%$ de sódio) e glutamato de sódio nos processos de industrialização dos alimentos, não tendo somente a função de agregar sabor, mas também para o controle do crescimento de micro-organismos em queijos, assim como para a preservação de vários outros alimentos (PERTSCHY; FRANCO, 2010). O consumo excessivo de sal também está associado ao câncer gástrico, podendo contribuir ainda, para o desenvolvimento de osteoporose.

Segundo Sarno et al. (2009), embora a maior parte do sódio disponível para consumo em todas classes de renda provenha do sal de cozinha e de condimentos à base desse sal (76,2\%), a fração proveniente de alimentos processados com adição de sal aumenta linear e intensamente com o poder aquisitivo domiciliar, representando $9,7 \%$ do total de sódio no quinto inferior da distribuição da renda per capita e $25,0 \%$ no quinto superior.

Nos países desenvolvidos, que contam com estimativas confiáveis sobre o consumo de sódio, a ingestão desse mineral tende a ultrapassar o limite máximo de $2 \mathrm{~g}$ (ou $5 \mathrm{~g}$ de sal) por pessoa por dia recomendado pela Organização Mundial da Saúde (SARNO et al., 2009), sendo a maior parte deste sódio proveniente de alimentos industrializados. A sua distribuição é universal, a sua ingestão 
varia de acordo com padrões culturais, dependendo da sua adição isolada ou associada com temperos para modular o sabor dos alimentos. O brasileiro consome em média de 2,8 a 5,0 g de sódio diariamente, sendo que a necessidade para humanos acima de 2 anos de idade varia de 0,3 a 0,5 g diários, recomendando-se que um adulto saudável não ultrapasse o limite de 2,4 g por dia (PERTSCHY; FRANCO, 2010).

A recomendação de consumo máximo diário de sal pela Organização Mundial de Saúde (OMS) é de menos de cinco gramas por pessoa. Dados do Instituto Brasileiro de Geografia e Estatística (IBGE) revelam, no entanto, que o consumo do brasileiro está em 12 gramas diários, valor que ultrapassa o dobro do recomendado (BESSA; SCHMIDT, 2012). Para Barreto et al. (2005) o consumo de sódio, de todas as fontes, deve ser limitado de maneira a reduzir o risco de doenças coronarianas e Acidente Vascular Encefálico (AVE). As evidências atuais sugerem que um consumo não superior a 70 mmol ou 1,7 $\mathrm{g}$ de sódio $(5 \mathrm{~g}$ de cloreto de sódio) por dia é benéfico para a redução da pressão arterial.

Em metanálise realizada por Feng e Graham (apud BARRETO et al., 2005) concluiu-se que a recomendação em torno de 5 a $6 \mathrm{~g} /$ dia de cloreto de sódio baseia-se mais no que é possível do que no nível cujo efeito positivo máximo pudesse ser alcançado. A análise do efeito dose resposta de ensaios clínicos de longa duração indicou que a redução de $3 \mathrm{~g} /$ dia de cloreto de sódio leva a uma queda na pressão de 3,6 a 5,6/1,9 a 3,2 $\mathrm{mmHg}$ (sistólica/diastólica) em indivíduos hipertensos; e de 1,8 a 3,5/0,8 a 1,8 mmHg em indivíduos normais. Esse efeito dobraria com a redução de $6 \mathrm{~g} /$ dia e triplicaria com a redução de 9g/dia. Segundo os autores, isso significaria, em uma estimativa conservadora, que a redução de $3 \mathrm{~g}$ do consumo diário de cloreto de sódio levaria a uma redução de $13 \%$ nos casos de AVE; e de $10 \%$ nas doenças isquêmicas do coração. Esse efeito dobraria com a redução de $6 \mathrm{~g}$ e triplicaria com a redução de 9g/dia. A recomendação atual para o nível de $6 \mathrm{~g} /$ dia teria efeito positivo na redução da hipertensão arterial, mas, ao longo prazo, não deveria ser considerada ideal. As informações disponíveis sobre consumo de sal provêm da indústria brasileira e indicam que a média de consumo de sal em 2000 era de 16,76 g por dia, seguindo uma tendência crescente. Prevendo-se que $10 \%$ desse total referem-se à alimentação animal e desperdício, pode-se inferir um consumo diário, por pessoa, de 15,08 g. Esse valor, comparado com a média de consumo dos países industrializados, que é de 8-9 g por dia, representa um dos níveis mais altos do mundo. Isso significa que a população do Brasil, em média, deveria diminuir o consumo do sal em dois terços, a 
fim de se aproximar do limite recomendável. Tendo em vista que a maioria do sal está contida nos alimentos industrializados, a conquista de uma redução substancial no consumo desse produto exigirá mudanças nas práticas de industrialização de alimentos.

O estudo desenvolvido por Tian et al. (apud MOLINA, 2003) na população chinesa, utilizando o método dietético (três recordatórios de 24h), identificou um consumo aproximado de $6 \mathrm{~g}$ de sódio na área urbana, sendo $53 \%$ provenientes de sal de adição, $17 \%$ de alimentos industrializados, $16 \%$ de molhos à base de soja e $6 \%$ de glutamato monossódico. Esse estudo sugere, ainda, a necessidade da redução de sódio no País, especialmente do sal adicionado no preparo das refeições por meio de estratégias populacionais visando ao controle da hipertensão arterial em todas as classes sociais.

No Canadá, o processo de redução do sódio em alimentos processados, iniciado em 2009, foi fortemente influenciado pela experiência britânica, adotando princípios e critérios semelhantes, com metas visando a redução do consumo per capita diário de sódio pela população para 2,3 g até 2016. O modelo canadense também propõe 0 estabelecimento de metas voluntárias e graduais com a indústria, a partir de reuniões técnicas e outros mecanismos de consulta e pactuação e baseadas em informações como o consumo alimentar da população, os níveis de sódio nos alimentos, a participação relativa das marcas e produtos no mercado e análises do processo produtivo, do comércio e da aceitação do consumidor (BRASIL. Ministério da Saúde, 2013).

O pão de queijo é um produto tradicionalmente brasileiro, obtido da mistura de polvilho (amido de mandioca) com água ou leite, queijo, ovos, sal e gordura, podendo variar o tipo de polvilho (doce, azedo ou a mistura destes). A produção do pão de queijo está em expansão, inclusive para o mercado externo (ZAVAREZE et al., 2009).

O pão de queijo não apresenta um padrão de qualidade estabelecido, não existindo uma tecnologia de produção, caracterização ou tipificação do produto. Existem duas formas principais de comercialização de pães de queijo prépreparados: as pré misturas, que são produtos nos quais se adicionam queijo, ovos, água entre outros ingredientes, dependendo da massa; e os pães de queijo congelados, que necessitam de aquecimento antes de serem consumidos (APLEVICZ; DEMIATE, 2007).

Além de ser uma fonte reconhecida de carboidratos, o pão de queijo também é um produto de panificação isento de glúten, o que o coloca como alimento alternativo para pacientes celíacos, intolerantes às proteínas do trigo. Sob a denominação de 
"pão de queijo" podem ser encontrados no mercado diferentes tipos de produtos com características bem distintas. Apesar de não haver uma tecnologia padronizada, o método de fabricação adotado pela grande maioria dos produtores de pão de queijo utiliza como ingredientes básicos polvilho doce e/ou azedo, queijo, óleo e ovos, seguindo um princípio básico de escaldamento do polvilho com água, óleo ou leite, amassamento com ovos, adição de queijo e assamento. O congelamento das massas de pão de queijo propiciou uma ampliação de mercado, interno e externo, que era pouco explorado para este produto (PEREIRA et al., 2004).

A produção de amidos modificados é uma alternativa que vem sendo desenvolvida há algum tempo com o objetivo de superar uma ou mais limitações dos amidos nativos e assim aumentar a utilidade deste polímero nas aplicações industriais (APLEVICZ; DEMIATE, 2007).

As razões que levam à utilização do amido modificado para desenvolvimento do produto em questão, segundo Silva et al. (2006), são que estes amidos são capazes de modificar as características de cozimento (gomificação); diminuir a retrogradação e a tendência das pastas em formarem géis; aumentar a estabilidade das pastas ao resfriamento e descongelamento; melhorar a textura das pastas ou géis; adicionar grupamentos hidrofóbicos e introduzir poder emulsificante. O uso do aditivo redutor de sódio na mistura para pão de queijo, torna o produto nutricionalmente mais saudável uma vez que possibilita a redução na adição de cloreto de sódio e promove uma diminuição do teor de sódio nestes produtos em comparação com os pães de queijo preparados de maneira convencional.

A presente pesquisa teve como objetivo o desenvolvimento de um pão de queijo com teor reduzido em sódio que satisfaça sensorialmente a expectativa dos consumidores e que ao mesmo tempo contribua para a redução do consumo de sódio, podendo ser consumido como parte de uma dieta saudável.

\section{MATERIAIS E MÉTODOS}

Tratou-se de um estudo original, de inferência observacional e transversal. A fabricação dos pães de queijo, bem como sua análise sensorial foi realizada, após aprovação pelo Comitê de Ética em Pesquisa (CEP) da UNOESTE, no CAAE 16853213.5.0000.5515, no laboratório de Pesquisa e Desenvolvimento da Podium Alimentos, utilizando os seguintes ingredientes: Amido Modificado para Pão de queijo, água, ovo pasteurizado, queijo muçarela, óleo de soja, leite em pó, margarina, sal e aditivo redutor de sódio.

A técnica de fabricação utilizada foi a convencional para o tipo de amido utilizado, 
sendo que a massa foi fracionada em porções de $30 \mathrm{~g}$ e moldadas manualmente no formato redondo. $\mathrm{Na}$ sequência submeteu-se $\mathrm{O}$ produto ao congelamento $(-18 \stackrel{\circ}{ } \mathrm{C})$ e após este processo o mesmo foi assado em forno elétrico por aproximadamente 25 minutos a $180^{\circ} \mathrm{C}$.

A caracterização sensorial foi realizada por 50 provadores voluntários, de ambos os gêneros, e faixa etária variável (acima de 18 anos) não treinados e com vínculo empregatício ao local em que o pão de queijo foi desenvolvido, após assinatura do Termo de Consentimento Livre e Esclarecido (TCLE). Os voluntários não foram identificados nas fichas de avaliação, sendo as mesmas depositadas em uma urna para o não constrangimento dos mesmos.

Foi realizado teste de aceitabilidade global do pão de queijo com redução de sódio, sendo utilizada para avaliação a escala hedônica estruturada de nove (09) pontos ancorados em extremos de "gostei extremamente" (9) e "desgostei muitíssimo" (1). Para comparação da aceitabilidade do pão de queijo com teor reduzido em sódio com o pão de queijo convencional, o mesmo teste foi aplicado para o pão de queijo convencional. O teste sensorial foi realizado de forma cega da seguinte maneira: ao provador voluntário foi apresentado primeiramente a amostra de $30 \mathrm{~g}$ de pão de queijo com teor reduzido em sódio, codificada com código de 3 dígitos (obtidos aleatoriamente). Juntamente, foi apresentada a ficha de avaliação sensorial. Após avaliação da aceitabilidade global (que envolve aparência, aroma, sabor e textura) desta amostra e o preenchimento da ficha de avaliação sensorial, o provador ingeriu água, com o intuito de lavar a boca para que não houvesse interferência do sabor da primeira amostra na conseguinte. Posteriormente foi apresentada a outra amostra de $30 \mathrm{~g}$ de pão de queijo convencional, também codificada com 3 dígitos aleatórios e a ficha de avaliação sensorial.

Os dados da análise sensorial foram tratados estatisticamente por análise de variância (ANOVA) e teste de Tukey para comparar a aceitabilidade do pão de queijo com redução de sódio e o pão de queijo convencional.

\section{RESULTADOS E DISCUSSÕES}

Ao avaliar os testes de aceitabilidade global aplicados no pão de queijo convencional e no produto com redução de sódio, observou-se que ambas formulações apresentaram notas médias similares $(7,85$ para o pão de queijo tradicional e 7,75 para o pão de queijo com teor reduzido em sódio). O resultado das análises estatísticas mostrou que esta diferença não foi significativa ao nível $5 \%$ de probabilidade $(p<0,05)$, conforme tabela1. 
Tabela 1. Notas médias da avaliação sensorial de pães de queijo tradicional e com teor de reduzido de sódio.

\begin{tabular}{lc}
\hline Pão de Queijo & Avaliação Sensorial \\
\hline Tradicional & $7,75 \mathrm{a}^{*}$ \\
Teor reduzido de sódio & $7,85 \mathrm{a}$ \\
\hline$*$ notas médias seguidas pela mesma letra na mesma coluna, não diferem estatisticamente ao \\
nível de 5\% de probabilidade $(\mathrm{p}<0,05)$ pelo teste $\mathrm{F}$.
\end{tabular}

Em estudo realizado por Vogel et al. (2011) foi verificado que em embutidos frescais a influência do teor de sódio na análise sensorial não alterou a aceitabilidade do produto quando mantido o limite de $60 \%$ de substituição de Cloreto de Sódio ( $\mathrm{NaCl})$ por Cloreto de Potássio (KCl). Contudo, a substituição de $80 \%$ de $\mathrm{NaCl}$ por $\mathrm{KCl}$ provocou sabor inaceitável nos embutidos.

Segundo Kishimoto et al. (2013) o produto Salona ${ }^{\mathrm{TM}}$ apresentou redução de até 45\% no teor de sódio em queijo muçarela sem que houvesse interferência na aceitação do produto. O Salona ${ }^{\mathrm{TM}}$ é um sal marinho natural com baixo teor de sódio, da ICL Performance Products/BKG Adicon que permite uma substituição de 25 a $50 \%$ de cloreto de sódio. Os minerais presentes neste sal marinho possuem naturalmente baixo teor de cloreto de sódio e são obtidos através da evaporação da água do Mar Morto em Israel. O produto Salona é constituído basicamente de $\mathrm{MgCl}_{2}, \mathrm{KCl}$ e $\mathrm{NaCl}$, sendo 31 $35 \%, 21-26 \%$ e $5 \%$, respectivamente.
Salovaara (1982) avaliou, na Finlândia, as características sensoriais de pães com diferentes níveis de substituição de cloreto de sódio $(5,10,20$ e $40 \%)$ por cloreto de potássio e sais de magnésio. A formulação controle (2\% de $\mathrm{NaCl}$ base farinha) apresentou semelhança à de pão francês. Testes triangulares mostraram que, com $20 \%$ de substituição por cloreto de potássio, não houve alterações no sabor; porém, com $40 \%$ de substituição deste sal, foi detectado sabor amargo.

\section{CONCLUSÃO}

Por meio do perfil sensorial foi possível observar que não houve diferenças sensoriais significativas entre o pão de queijo com redução de sódio e o produto convencional, tendo este, boa aceitabilidade por parte dos provadores. Mediante os resultados obtidos conclui-se que o produto desenvolvido pode fazer parte de uma dieta saudável e agradar sensorialmente os consumidores. 


\section{AGRADECIMENTOS}

Este trabalho contou com a colaboração, auxílio técnico e de infra estrutura da Comercial Anhumaí - Podium Alimentos.

\section{REFERÊNCIAS}

APLEVICZ, K.S.; DEMIATE, I.M. Análises físicoquímicas de pré-misturas de pães de queijo e produção de pães de queijo com adição de okara. Ciênc. Agrotec., v.31, n.5, p.14161422, 2007. http://dx.doi.org/10.1590/S1413$\underline{70542007000500022}$

BARRETO, S.M.; PINHEIRO, A.R.O.; SICHIERI, R.; MONTEIRO, C.A.; BATISTA FILHO, M.; SCHIMIDT, M.I.; LOTUFO, P.; ASSIS, A.M.; GUIMARÃES, V.; RECINE, E.G.I. G.; VICTORA, C.G.; COITINHO, D.; PASSOS, V.M.A. Análise da estratégia global para alimentação, atividade física e saúde, da Organização Mundial da Saúde. Epidemiol. Serv. Saúde., v.14, n.1, p.41-68, 2005.

BESSA, S; SCHMIDT, F. Acordo para redução de sódio inclui novos alimentos. Brasília: Ministério da Saúde, 2012. Disponível em: <http://portalsaude.gov.br/portalsaude/noti cia/6829/162/acordo-para-redução-de-sodioinclui-novos-alimentos.html. Acesso em: 10 set. 2013.

BRASIL. Ministério da Saúde. Plano de redução de sódio em alimentos processados. $2013 . \quad$ Disponível em: <http://www.abia.org.br/anexos/Criteriospar amonitoramentoeavaliacaodoPlano27jan.pdf >. Acesso em: 19 fev. 2014.

KISHIMOTO, G.B.; LIMA, R.S.; ROSA, C.M.C.; MOREIRA, R.O.; PERRONE, I.T.; CARVALHO, A. F. Desenvolvimento de queijo mussarela com baixo teor de sódio. 2013. Disponível em: <http://www.milkpoint.com.br/mypoint/224 299/p_desenvolvimento_de_queijo_mussare la_com_baixo_teor_de_sodio_mussarela_red ucao_de_sodio_queijos_inovacao_inovaleite _5205.aspx>. Acesso em: 09 ago. 2014.

MOLINA, M.C.B.; CUNHA, R.S.; HERKENHOFF, L.F.; MILL, J.G. Hipertensão arterial e consumo de sal em população urbana. Rev. Saúde Pública, v.37, n.6, p. 743-750, 2003.

ORVALHO, R.J.S. Redução do teor de sódio em fiambre. Implicações tecnológicas, organolépticas e de prazo de validade. 2010. 89f. Dissertação (Mestrado em Medicina Veterinária) - Universidade Técnica de Lisboa, Faculdade de Medicina Veterinária, Lisboa.

PEREIRA, J.; CIACCO, CF.; VILELA, E.R.; PEREIRA, R.G.F.A. Função dos ingredientes na consistência da massa e nas características do pão de queijo. Ciênc. Tecnol. Aliment., v.24, n.4, p. 494-500, 2004. http://dx.doi.org/10.1590/S0101$\underline{20612004000400003}$

PERTSCHY, P.; FRANCO, S. Comparação do teor de sódio nos alimentos convencionais, ligth e diet pela rotulagem dos produtos. Guarapuava: Universidade Estadual do Centro-Oeste, 2010.

SALOVAARA, H. Sensory limitations to replacement of sodium with potassium and magnesium in bread. Cereal Chemistry, v.59, n.5, p.427-430, 1982. Disponível em: $<$ http://www.aaccnet.org/publications/cc/ba ckissues/1982/Documents/chem59_427.pdf> . Acesso em: 09 ago. 2014.

SARNO, F.; CLARO, R.M.; LEVY, R.B.; BANDONI, D.H.; FERREIRA, S.R.G.; MONTEIRO, C.A. Estimativa de consumo de sódio pela população brasileira, 2002-2003. Rev. Saúde Pública, v.43, n.2, p.219-225, $2009 . \quad$ http://dx.doi.org/10.1590/S003489102009005000002 
SILVA, G.O.; TAKIZAWA, F.F.; PEDROSO, R.A.; FRANCO, C.M.L.; LEONEL, M.; SARMENTO, S.B.S.; DEMIATE, I.M. Características físicoquímicas de amidos modificados de grau alimentício comercializados no Brasil. Ciênc. Tecnol. Aliment., v.26, n.1, p. 188-197, 2006. http://dx.doi.org/10.1590/S0101$\underline{20612006000100030}$

VOGEL, C.C.; PAZUCH, C.M.; SARMENTO, C.M.P.; BACK, L.; SECCO, T.H. Desenvolvimento de salsicha com teor reduzido (sal light). Revista Ciências Exatas e Naturais, v.13, n.3, Edição Especial, 2011.

ZAVAREZE, E.R.; STORCK, C.R.; PEREIRA, J.M.; GULARTE, M.A.; DIAS, A.R.G. Elaboração de pão de queijo com substituição do amido de mandioca por amido de batata doce (Ipomoea batata) submetido a diferentes processos de secagem. Braz. J. Food Technol., v.12, n. 1, p. 68-76, jan./mar. 2009. http://dx.doi.org/10.4260/BJFT20094908000

$\underline{09}$ 